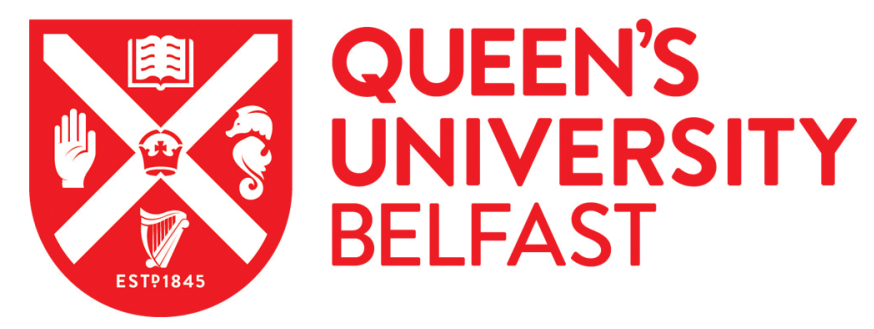

\title{
A comparison of choline:urea and choline:oxalic acid deep eutectic solvents at $338 \mathrm{~K}$
}

Gilmore, M., Moura, L., Turner, A., Swadzba-Kwasny, M., Callear, S., McCune, J. A., Scherman, O. A., \& Holbrey, J. (2018). A comparison of choline:urea and choline:oxalic acid deep eutectic solvents at $338 \mathrm{~K}$. Journal of Chemical Physics, 148, [193823]. https://doi.org/10.1063/1.5010246

Published in:

Journal of Chemical Physics

Document Version:

Peer reviewed version

Queen's University Belfast - Research Portal:

Link to publication record in Queen's University Belfast Research Portal

Publisher rights

Copyright AlP Publishing, This work is made available online in accordance with the publisher's policies. Please refer to any applicable terms of use of the publisher.

\section{General rights}

Copyright for the publications made accessible via the Queen's University Belfast Research Portal is retained by the author(s) and / or other copyright owners and it is a condition of accessing these publications that users recognise and abide by the legal requirements associated with these rights.

Take down policy

The Research Portal is Queen's institutional repository that provides access to Queen's research output. Every effort has been made to ensure that content in the Research Portal does not infringe any person's rights, or applicable UK laws. If you discover content in the Research Portal that you believe breaches copyright or violates any law, please contact openaccess@qub.ac.uk. 
Choline-urea and choline-oxalic acid DES

A comparison of choline:urea and choline:oxalic acid deep eutectic solvents at $338 \mathrm{~K}$ Mark Gilmore, ${ }^{1}$ Leila M. Moura, ${ }^{1}$ Adam H. Turner, ${ }^{1}$ Małgorzata Swadźba-Kwaśny, ${ }^{1}$ Samantha K. Callear, ${ }^{2}$ Jade A. McCune, ${ }^{3}$ Oren A. Scherman, ${ }^{3}$ and John D Holbrey ${ }^{1, a)}$

1) School of Chemistry and Chemical Engineering, The Queen's University of Belfast, Belfast, BT9 5AG, Northern Ireland, UK. Tel: +44 (0) 2890974836

2) ISIS, Rutherford Appleton Laboratory, Harwell Science $\&$ Innovation Campus, Didcot, Oxfordshire, OX11 ODE, UK

3) Melville Laboratory for Polymer Synthesis Department of Chemistry, University of Cambridge Lensfield Road, Cambridge, CB2 1EW, $U K$

(Dated: February 6, 2018)

1:2 Choline chloride:urea and 1:1 choline chloride:oxalic acid deep eutectic solvents (DES) are compared at $338 \mathrm{~K}$ using liquid-phase neutron diffraction with $\mathrm{H} / \mathrm{D}$ isotopic substitution to obtain differential neutron scattering cross sections and fitting of models to the experimental data using Empirical Potential Structure Refinement (EPSR). In comparison to the previously reported study of choline chloride:urea at $303 \mathrm{~K}$, we observed significant weakening and lengthening of choline- $\mathrm{OH} \cdots \mathrm{Cl}^{-}$and choline-OH $\cdots$ hydrogen-bond acceptor correlations.

Keywords: DES, Choline chloride, oxalic acid, urea, neutron scattering, EPSR

a)Electronic mail: j.holbrey@qub.ac.uk 


\section{INTRODUCTION}

The term Deep Eutectic Solvent (DES) popularised by Abbott et al. ${ }^{1}$ is most commonly used to describe low melting liquids formed by combining organic salts such as choline chloride with urea ${ }^{2}(\mathrm{ChCl}: \mathrm{U})$ or other hydrogen-bond donor components. These can include carboxylic acids ${ }^{3}$ and alcohols. ${ }^{4}$

Choline-based DES can exhibit remarkable melting point depressions, ${ }^{5,6}$ for example $\mathrm{ChCl:U} \mathrm{1:2} \mathrm{has} \mathrm{a} \mathrm{melting} \mathrm{point} 178 \mathrm{~K}$ lower than that of the two components and it was formerly thought that formation of complex anions (for example $\left[(\text { urea })_{2} \mathrm{Cl}\right]^{-}$) through hydrogen bond donor (HBD) to anion hydrogen-bonding was the driving force for liquid formation. ${ }^{6-8}$

Recent neutron scattering studies ${ }^{9-12}$ and simulation ${ }^{13-17}$ have revealed a much more complex picture of the liquid structures ${ }^{18}$ with competing strong and weak Coulombic and hydrogen-bonding interactions between all the liquid components. However, the details of the relative contributions of different interactions to the structure and experimental data on which to build this understanding is only slowly being developed. Notably, Ashworth et al. ${ }^{14}$ have discussed the need to consider the complete range of hydrogen-bonding and Coulombic interactions possible, and the potential to form both complex anions (e.g. urea $[\mathrm{Cl}]^{-}$) and cations (e.g. urea $\left.[\text { choline }]^{+}\right)$.

Most recently using INS and vibration spectroscopy, Araujo et al. ${ }^{19}$ have argued that the interplay of soft and strong interactions in $\mathrm{ChCl}$ : $\mathrm{U}$ confers flexibility to newly formed hydrogen-bonding networks allowing the ensemble to remain liquid at room temperature. It was noted that short choline-OH…Cl correlations (at 2.1 A) reported by Hammond, Bowron, and Edler ${ }^{11}$ with similar separations to those found in the crystal structure of choline chloride are contrary to expectation. Further more, vibrational analysis revealed that choline exists predominantly in the gauche conformer in the DES with a small trans population that is indicative of freer but still restricted rotation of the $\mathrm{C}-\mathrm{C}$ bond in the liquid state than in the solid and that urea moves from the solid state $\mathrm{sp}^{2}$ planar structure to one with the nitrogen sites adopting a partially pyramidal $\mathrm{sp}^{3}$ conformation. This loss of planarity is a direct response to the fluctuating and flexible hydrogen bond network in the DES with the urea engaging in a wide number of intermolecular contacts with varying strengths.

The chloride ion environment in the 1:1 choline:malic acid (ChCl:Mal) DES has been described as more robust than in $\mathrm{ChCl}: \mathrm{U}$ with respect to dissociation forming acid-acid 
Choline-urea and choline-oxalic acid DES

clusters. ${ }^{12}$ This was rationalised in terms of stronger chelation of chloride by malic acid and appears consistent with QENS $^{9,12}$ measurements that show lower chloride anion mobility compared to choline cations suggesting greater involvement of chloride in hydrogen-bonding networks.

These descriptions of the DES liquid network, with correlations between all the components is analogous to that also found mixtures containing ionic liquids, for example ionic

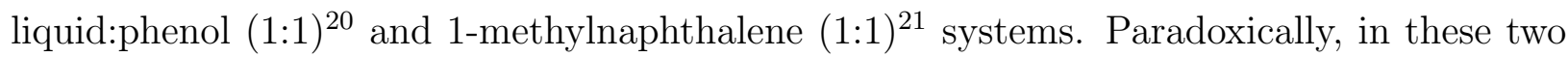
examples, the former is a eutectic mixture whereas the latter forms a stable peritectic 1:1 complex. As interest in DES-forming systems continues to increase, there is a need to obtain additional experimental structural data to understand how changes in the nature of the HBD components affects structure and properties, especially the effect of temperature, taking into account the growing interest in using DES for ionothermal materials synthesis. ${ }^{22}$

Here, we present experimental neutron scattering data performed at $338 \mathrm{~K}$ with EPSR structure analysis to compare $\mathrm{ChCl:U}$ and choline chloride:oxalic acid 1:1 (ChCl:Ox) in order to (i) evaluate thermal effects compared to the structure of $\mathrm{ChCl:U}$ reported at $303 \mathrm{~K}^{11}$ and (ii) to investigate $\mathrm{ChCl}: \mathrm{Ox}$ which is crystalline at room temperature ${ }^{3}$ and is of interest for metal oxide dissolution.

\section{EXPERIMENTAL}

Hydrogenated choline chloride, anhydrous oxalic acid, urea and urea- $\mathrm{D}_{4}$ were purchased from Sigma-Aldrich. Choline chloride- $D_{9}\left(\left[\left(\mathrm{CD}_{3}\right)_{3} \mathrm{~N}\left(\mathrm{CH}_{2}\right)_{2} \mathrm{OH}\right] \mathrm{Cl}\right)$ was purchased from CDN Isotopes (QMX Laboratories, Thaxted, UK) and Cambridge Isotopes (Goss Scientific Instruments Limited, Crewe, UK). Anhydrous oxalic acid- $\mathrm{D}_{2}$ was prepared by H/D exchange from oxalic acid with $\mathrm{D}_{2} \mathrm{O}(3 \times)$ followed by freeze-drying under vacuum.

Isotopically substituted samples were made by mixing the appropriate deuteriated or protiated choline chloride with urea (1:2 molar ratio) and oxalic acid (1:1 molar ratio). Samples were prepared under an inert atmosphere, heating at $333 \mathrm{~K}$ until homogeneous liquids were produced. On cooling to room temperature all the DES samples solidified. The ChCl:U DES melted at $304 \mathrm{~K}$, consistent with literature for $d r y \mathrm{ChCl:U}$ DES. ${ }^{23}$ Melting points fo the crystalline ChCl:Ox DES ranged from ca. 323-333 K depending on the isotopomeric substitution and contrast with the reported solidification temperature of $307 \mathrm{~K} .{ }^{3}$ The higher 
Choline-urea and choline-oxalic acid DES

Table I. Initial Lennard-Jones $(\epsilon$ and $\sigma)$ and charge $(q)$ parameters used for the reference potential of the Empirical Potential Structure Refinement model for both systems derived from the literature, and OPLS-AA.

$\overline{\text { Atom type } \epsilon / \mathrm{kJ} \mathrm{mol}^{-1} \sigma / \AA \quad q / \mathrm{e}}$

\section{Choline Chloride}

$\begin{array}{llll}\text { Cl } & 0.80 & 4.0 & -1.0000 \\ \mathrm{~N} & 0.70 & 3.2 & -0.15456 \\ \mathrm{CM} & 0.80 & 3.7 & 0.10974 \\ \mathrm{CE} & 0.80 & 3.7 & 0.07411 \\ \mathrm{HM} & 0.00 & 0.0 & 0.05244 \\ \mathrm{CT} & 0.80 & 3.7 & 0.16201 \\ \mathrm{HE} & 0.00 & 0.0 & 0.05244 \\ \mathrm{HT} & 0.00 & 0.0 & 0.05244 \\ \mathrm{OH} & 0.65 & 3.1 & -0.3126 \\ \mathrm{HO} & 0.00 & 0.0 & 0.22008\end{array}$

Oxalic acid

$\begin{array}{llll}\text { CA } & 0.80 & 3.7 & 0.68306 \\ \text { OA1 } & 0.65 & 3.1 & -0.53552 \\ \text { OA2 } & 0.65 & 3.1 & -0.54506 \\ \text { HA } & 0.00 & 0.0 & 0.44452\end{array}$

Urea

\begin{tabular}{llll}
$\mathrm{CU}$ & 0.80 & 3.7 & 0.40060 \\
$\mathrm{OU}$ & 0.65 & 3.1 & -0.41760 \\
$\mathrm{NU}$ & 0.70 & 3.2 & -0.45240 \\
$\mathrm{HU}$ & 0.00 & 0.0 & 0.23045 \\
\hline \hline
\end{tabular}


Choline-urea and choline-oxalic acid DES

Table II. Intramolecular bond distance $(\AA)$ and bond-angle $\left(^{\circ}\right)$ constraints used to define the basic structure of the components in the initial EPSR simulation model. Oxalic acid was fixed to a planar conformation by defining O-C-C-O dihedral restraints.

Bond $/ \AA$ Bond Angle $/{ }^{\circ}$

\section{Choline Chloride}

CM-HM 1.08 HM-CM-HM 108.5

CM-N $\quad 1.51$ HM-CM-N $\quad 108.5$

$\mathrm{N}-\mathrm{CE} \quad 1.56 \mathrm{CM}-\mathrm{N}-\mathrm{CM} \quad 108.5$

CE-HE 1.08 CM-N-CE 110.0

CE-CT 1.46 N-CE-HE 108.5

CT-HT 1.08 N-CE-CT 114.6

CT-OH 1.44 HE-CE-HE 108.5

$\mathrm{OH}-\mathrm{HO} \quad 0.97 \mathrm{HE}-\mathrm{CE}-\mathrm{CT} \quad 108.5$

CE-CT-HT 108.5

CE-CT-OH 112.6

HT-CT-HT 108.5

$\mathrm{OH}-\mathrm{CT}-\mathrm{HT} \quad 108.5$

CT-OH-HO 118.0

\section{Oxalic acid}

OA1-CA 1.22 OA1-CA-OA2 119.5

OA2-CA 1.35 CA-OA2-HA 115.6

CA-CA 1.54 OA1-CA-CA 124.3

OA2-HA 0.95 OA2-CA-CA 116.3

\begin{tabular}{lll} 
Urea & & \\
OU-CU & 1.26 OU-CU-NU & 119.9 \\
NU-CU & 1.39 CU-NU-HU & 120.1 \\
NU-HU & 0.99 HU-NU-HU & 119.7 \\
\hline \hline
\end{tabular}




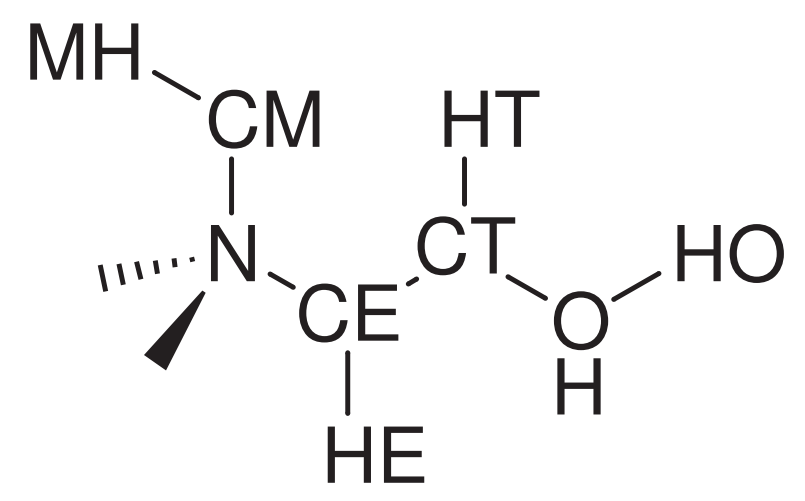

\section{Choline}

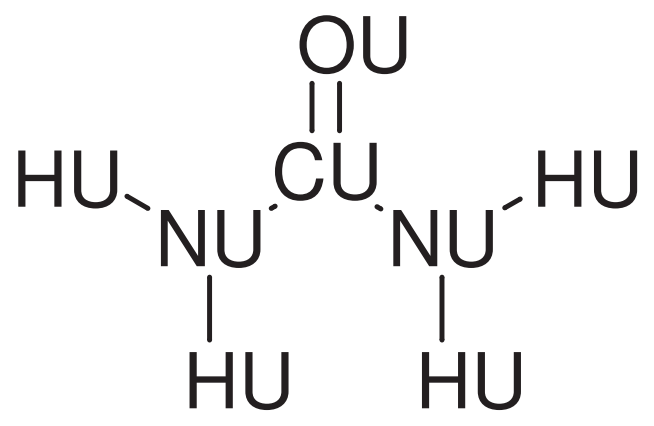

Urea

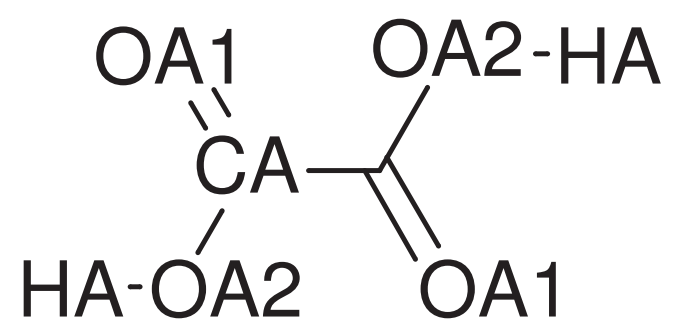

Oxalic Acid

Figure 1. Atom types assigned to the choline, urea and oxalic acid molecules used in the EPSR simulation of diffraction data.

values found here are ascribed to the carefully controlled absence of water in the samples, consistent with reports that recognise that advantitious water in hydroscopic DES causes melting point supression. ${ }^{23}$

Seven isotopic contrast samples were prepared for ChCl:Ox with compositions of H:D, D:H, H:H, D:D, H/D:H/D, H/D:D, and D:H/D (choline chloride:hydrogen bond donor), where H/D signifies equimolar mixtures of the hydrogenation and deuteriated components. For ChCl:U, four compositions (H:H, D:D, H:D, and D:H) were examined.

Neutron scattering data were collected at the ISIS pulsed neutron and muon source at 
the Rutherford Appleton Laboratory, UK using the SANDALS (ChCl:Ox) and NIMROD (ChCl:U) spectrometers. Each sample was contained in 'null scattering' $\mathrm{Ti}_{0.68} \mathrm{Zr}_{0.32}$ flat plate cells with internal geometries of $1 \times 35 \times 35 \mathrm{~mm}$, with a wall thickness of $1 \mathrm{~mm}$. During measurements, the cell was maintained at a temperature of $338 \mathrm{~K}$ using a recirculating heater (Julabo FP50). Measurements were made on each of the empty sample holders, the empty spectrometer, and a $3.1 \mathrm{~mm}$ thick vanadium standard sample for the purposes of instrument calibration and data normalisation.

Data reduction was performed using GUDRUN, ${ }^{24}$ to produce a differential scattering cross section for each experimental sample. The experimental sample densities and scattering levels were consistent with the actual isotopic compositions of the samples. Calibration and background subtraction for single atom scattering was made to produce a differential scattering cross section for each sample. Data from the neutron diffraction experiments was analysed using the Empirical Potential Structure Refinement (EPSR) program. ${ }^{25,26}$

The experimental total structure factors, $\mathrm{F}(Q)$, were extracted from the neutron scattering data for each of the isotopically distinct samples at each composition. These were used to build and refine three dimensional models of the liquid structure consistent with the experimental data using EPSR for each DES. By comparing the differences between calculated and experimental structure factors in $Q$-space for data sets, an empirical perturbation potential is determined. This is combined with the reference potential and used as the new potential for simulations, iteratively driving the simulation model towards agreement with experimental data.

The EPSR refinements were performed using 400 choline chlorides and 800 urea molecules (ChCl:U 1:2), and 500 choline chloride ion pairs and 500 oxalic acid molecules (ChCl:Ox 1:1). The simulations were performed using cubic boxes of dimension 53.51 and $54.06 \AA$ respectively. Models were refined against the experimental data over the full data range $(Q$ $\left.=0.1-50 \AA^{-1}\right)$. Within the EPSR simulation, initial potentials and interatomic distance constraints used to define the basic molecular geometries were obtained from MOPAC with the AM1 model. Atom types in each system were defined based on their unique positions in the molecular skeletons, as shown in Figure 1, and full rotational flexibility was enabled in the model. The full parameters of the reference potential used are given in Table I and the interatomic distance and angular constraints used to define the basic molecular geometries are summarised in Table II. 
Choline-urea and choline-oxalic acid DES

Table III. Position of the first peak in the centre of mass RDFs for 3. Results from Hammond, Bowron, and Edler ${ }^{11}$ for $\mathrm{ChCl:U}$ are shown in parentheses.

\begin{tabular}{llc}
\hline \hline & \multicolumn{2}{c}{ Peak Position $/ \AA$} \\
\cline { 2 - 3 } Pair & ChCl:U & ChCl:Ox \\
\hline Choline-Choline & $6.4(6.3)$ & 6.3 \\
Choline-Cl & & 4.5 \\
Choline-HBD & $4.6(4.2)$ & 5.7 \\
HBD-HBD & $5.4(5.5)$ & 5.0 \\
HBD-Cl ${ }^{-}$ & $4.3(4.4)$ & 3.7 \\
\hline \hline
\end{tabular}

Simulations were allowed to equilibrate for at least 2500 cycles before applying the empirical potential, then were equilibrated over ca. 10000 cycles before accumulating and averaging data. The total numeric density of the simulation box corresponded to the experimentally determined molecular densities of the fully protiated materials. Centre of mass radial distribution functions (RDFs) were calculated using the SHARM routines within EPSR.

\section{RESULTS AND DISCUSSION}

\section{Experimental neutron diffraction data and fits}

Experimental neutron diffraction data collected at $338 \mathrm{~K}$ for $\mathrm{ChCl:U}(1: 2)$ and $\mathrm{ChCl:Ox}$ (1:1) was modelled with EPSR, refining against the experimental data from each set of H/D isotopically distinct samples. Data and the corresponding fits from the EPSR simulation (Figure 2) show good consistency between the experimental and the EPSR derived models. The greatest source of discrepancy between experimental data sets and EPSR fits were found at $Q<1 \AA$ where corrections for inelastic scattering by hydrogen are most difficult. ${ }^{27}$ In addition, the poorest convergence of experimental and fitted scattering data was found for the H:D samples containing protiated choline and either deuteriated urea or oxalic acid.

Centre of mass RDFs for choline, $\mathrm{Cl}^{-}$, and the HBD component (urea or oxalic acid) in the two DES are shown in Figure 3 with the corresponding peak maxima for the first shells shown in Table III. These correlation distances are comparable to the equivalent 
Choline-urea and choline-oxalic acid DES
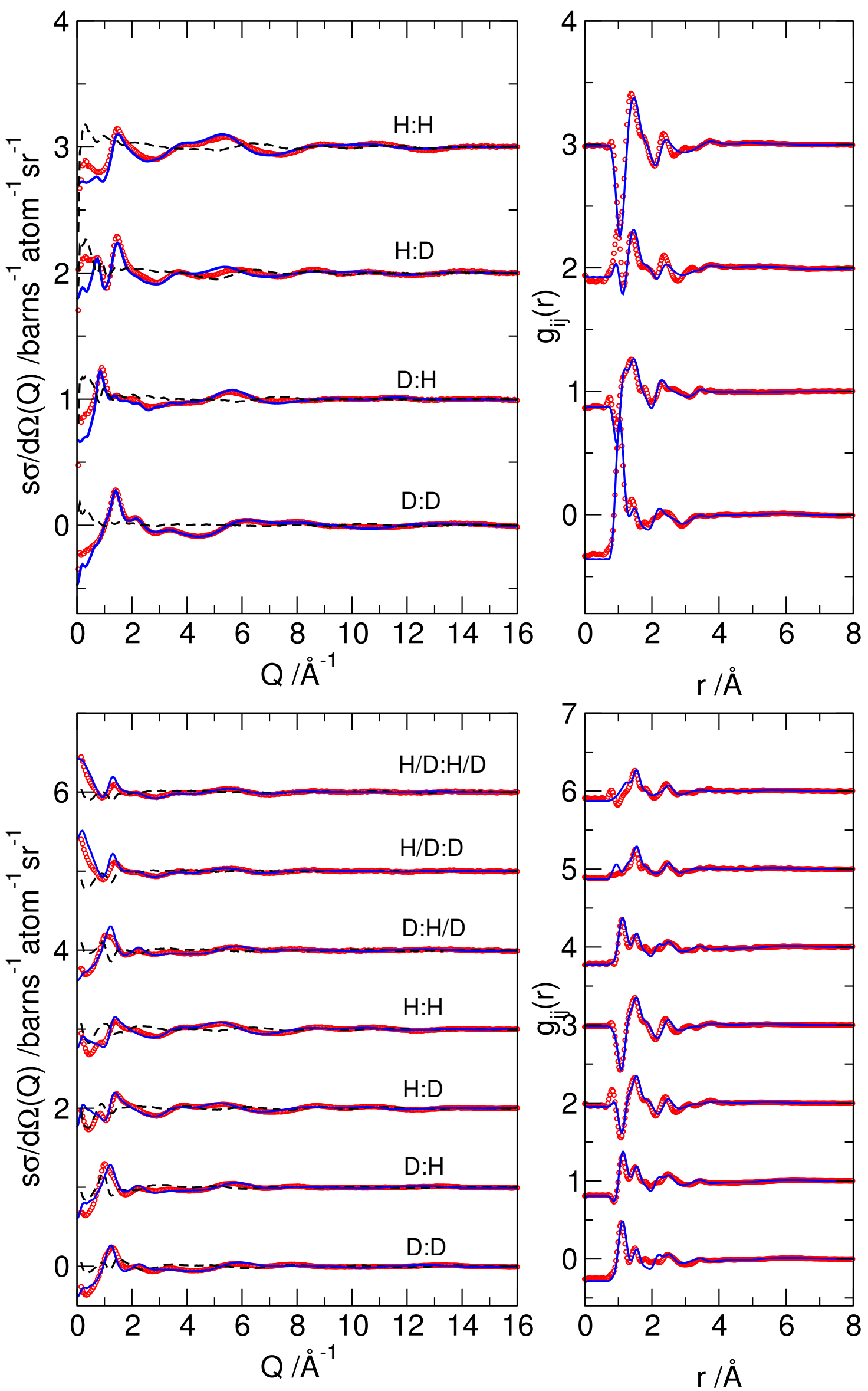

9

Figure 2. Structure factors for ChCl:U (top) and ChCl:Ox (bottom). For each DES, experimental 
site-site pRDFs reported at $303 \mathrm{~K}^{11}$ and $\mathrm{ChCl}: \mathrm{Mal}^{12}$ and, in general, with computational experimental results. ${ }^{16,28}$ Both $\mathrm{ChCl}: \mathrm{U}$ and $\mathrm{ChCl}$ :Ox show similar centre of mass RDFs with comparable correlation peak positions and shapes. The notable exceptions are correlations to the HBD sites (urea or oxalic acid) where the peak in the respective RDFs occur at shorter distances for urea than oxalic acid. This can be rationalised in terms of the relative sizes of the two HBD. Strong correlations are clearly evident between the HDB molecules and both chloride and other HBD molecules. In both $\mathrm{ChCl:U}$ and $\mathrm{ChCl}$ :Ox, the choline- $\mathrm{HBD}$ peak (5.4-6.0 $\AA$ ) occurs at longer distances than the choline-chloride correlation (4.5-5.0 $\AA)$ but at a shorter distance than the corresponding choline-choline correlation (6.3-6.4 $\AA$ ) indicating that there is intercalation of the molecular HBD components into the ionic lattice.

Choline-chloride and choline-choline RDFs for both DES show similar profiles, revealing an alternating lattice arrangement of charged ions that resembles the structure of imidazolium chloride ionic liquids. ${ }^{29}$ In both DES, the choline-chloride RDF shows a single broad maximum for close contact correlations at 4.5-4.6 $\AA$. This contrasts with the reported room temperature data for $\mathrm{ChCl}: \mathrm{U}^{11}{ }^{11}$ where a double peak feature (sharp maximum at $c a .4 .0$ $\AA$ and a broader shoulder around $5.2 \AA$ ) was reported. The peak at $4.0 \AA$ was assigned to strong hydrogen-bonding choline-hydroxyl to chloride association and the longer distance component to association of chloride ions with the trimethylammonium region of the choline cation. These differences appear to indicate a merging of the two ambient temperature correlations with broadening due to the higher temperature lattice expansion.

Site-site partial radial distribution functions (pRDFs) were extracted from the EPSR refinements in order to further analyse the individual interactions. Principle correlations of interest are shown in Figure 4. The positions of the first peak $\left(r_{\max }\right)$ in the pRDF and corresponding coordination numbers are shown in Table IV.

\section{Structure of ChCl:U DES}

CE-CE and CE-Cl pRDF correlations (Figure 4) have coordination numbers of 6.94 and 3.75 which compare to values of 6.74 and 4.35 at $303 \mathrm{~K}^{11}$ These pRDFs correspond to the choline-choline and choline-chloride centre of mass RDFs in Figure 3 and indicate that the general liquid structure of the DES is retained as the temperature is increased. However, as with the choline-chloride COM RDF as noted above, the shapes of the $\mathrm{Cl}-\mathrm{N}$ and $\mathrm{Cl}-\mathrm{CE}$ 
Choline-urea and choline-oxalic acid DES

Table IV. Coordination numbers calculated for the principle partial RDFs shown in Fig. 4 for ChCl:U (1:2) and ChCl:Ox 1:1 DES. Mean coordination numbers $N_{\text {coord }}$ were determined to the first minima $\left(r_{\min }\right)$ after the primary correlation peak $\left(r_{\max }\right)$ in the pRDF.

\begin{tabular}{|c|c|c|c|c|c|}
\hline $\mathrm{pRDF}$ & $r_{\max }\left(r_{\min }\right) / \AA$ & $N_{\text {coord }}$ & $\mathrm{pRDF}$ & $r_{\max }\left(r_{\min }\right) / \AA$ & $N_{\text {coord }}$ \\
\hline & \multicolumn{2}{|c|}{ ChCl:U 1:2 } & & \multicolumn{2}{|c|}{ ChCl:Ox 1:1 } \\
\hline \multicolumn{6}{|c|}{ Choline-Choline } \\
\hline $\mathrm{N}-\mathrm{N}$ & $6.2(8.2)$ & $4.82 \pm 1.23$ & & $6.1(8.2)$ & $7.07 \pm 1.69$ \\
\hline $\mathrm{CE}-\mathrm{CE}$ & $6.4(9.0)$ & $6.94 \pm 1.36$ & & $6.3(9.0)$ & $9.17 \pm 1.85$ \\
\hline $\mathrm{CE}-\mathrm{N}$ & $5.4(8.5)$ & $6.69 \pm 1.34$ & & $5.4(8.5)$ & $8.68 \pm 1.77$ \\
\hline $\mathrm{N}-\mathrm{CT}$ & $5.3(7.0)$ & $3.88 \pm 1.38$ & & $5.3(7.0)$ & $4.80 \pm 1.51$ \\
\hline $\mathrm{N}-\mathrm{OH}$ & $4.9(5.8)$ & $2.26 \pm 1.02$ & & $4.8(5.8)$ & $2.78 \pm 1.11$ \\
\hline $\mathrm{N}-\mathrm{HO}$ & $4.6(6.0)$ & $2.64 \pm 1.16$ & & $4.6(6.0)$ & $3.16 \pm 1.25$ \\
\hline $\mathrm{OH}-\mathrm{HO}$ & $3.2(4.0)$ & $0.67 \pm 0.77$ & & $3.1(4.0)$ & $0.80 \pm 0.79$ \\
\hline
\end{tabular}

\section{Choline-Chloride}

$\begin{array}{lllll}\mathrm{N}-\mathrm{Cl} & 4.7(5.5) & 2.40 \pm 0.89 & 4.7(5.5) & 3.25 \pm 1.07 \\ \mathrm{CE}-\mathrm{Cl} & 3.7(6.8) & 3.75 \pm 1.00 & 3.6(6.8) & 4.73 \pm 1.21 \\ \mathrm{Cl}-\mathrm{HM} & 2.8(4.0) & 5.75 \pm 2.30 & 2.8(4.0) & 7.85 \pm 2.68 \\ \mathrm{Cl}-\mathrm{HE} & 2.8(4.0) & 1.36 \pm 1.25 & 2.8(4.0) & 1.85 \pm 1.23 \\ \mathrm{Cl}-\mathrm{HT} & 2.8(4.0) & 1.35 \pm 1.24 & 2.8(4.0) & 1.85 \pm 1.19 \\ \mathrm{Cl}-\mathrm{HO} & 2.8(4.0) & 0.80 \pm 0.84 & 2.8(4.0) & 0.92 \pm 0.71\end{array}$

\section{Choline-Urea}

Choline-Oxalic acid

$\begin{array}{lcclll}\text { N-CU } & 5.2(7.8) & 10.88 \pm 1.94 & \text { N-CA } & 5.2(7.7) & 11.91 \pm .75 \\ \text { N-NU } & 4.9(7.5) & 19.83 \pm 3.71 & \text { CE-CA } & 4.5(8.2) & 14.31 \pm 4.73 \\ \text { N-OU } & 4.7(5.6) & 9.12 \pm 2.35 & \text { OA1-HO } & 2.7(4.0) & 0.57 \pm 0.74 \\ \text { OU-HO } & 2.8(4.0) & 0.49 \pm 0.66 & \text { OA2-HO } & 2.7(4.0) & 0.59 \pm 0.72 \\ \text { OU-HM } & 2.8(3.5) & 2.75 \pm 1.65 & \text { OA1-HM } & 2.7(4.0) & 4.33 \pm 2.40 \\ \text { OU-HE } & 2.8(3.5) & 0.56 \pm 0.76 & \text { OA2-HM } & 2.7(4.0) & 4.15 \pm 2.33 \\ \text { OU-HT } & 2.8(3.5) & 0.58 \pm 0.82 & \text { OA1-HE } & 2.7(4.0) & 0.97 \pm 1.10 \\ \text { OH-HU } & 2.0(4.0) & 3.37 \pm 2.09 & \text { OA2-HE } & 2.7(4.0) & 0.93 \pm 1.08 \\ & & 11 & \text { OA1-HT } & 2.7(4.0) & 0.93 \pm 1.06 \\ & & & \text { OA2-HT } & 2.7(4.0) & 0.98 \pm 1.06\end{array}$


peaks differ.

The $\mathrm{N} \cdots \mathrm{Cl}$ correlation appears as a peak at $4.7 \AA$ (coordination number 2.40) with a small shoulder at $4.2 \AA$. This correlation distance is $c a$. $0.5 \AA$ longer than that found by Hammond, Bowron, and Edler ${ }^{11}$ at $303 \mathrm{~K}$ and the correlation length of $3.9 \AA$ with a weaker shoulder at $4.8 \AA$ determined from small cluster DFT calculations. ${ }^{16}$ The longer correlation length found here can be interpreted in terms of increased mobility of the choline cation, however the variations in the correlation profile can only be explained by an overall change in the orientational association between choline and chloride ions. The $\mathrm{CE} \cdots \mathrm{Cl}$ correlation has a pronounced bimodal distribution, with two peaks at $3.6 \AA$ and $4.7-5.7 \AA$ sitting either side of the $\mathrm{N} \cdots \mathrm{Cl}$ correlation. Similar profiles are present for all three $\mathrm{C}(\mathrm{x}) \cdots \mathrm{Cl}$ correlations from choline to chloride with a first peak in the range $3.55-3.65 \AA$ and corresponding $\mathrm{H} \cdots \mathrm{Cl}$ correlations at $2.8 \AA$.

Two different choline-chloride interaction motifs have been previously reported; strong hydrogen-bonding between the choline-hydroxyl group to chloride and Coulombic charge association of chloride ions with the trimethylammonium cation. Hammond, Bowron, and Edler ${ }^{11}$ identified choline-hydroxyl to chloride association as a significant contributor to structural ordering at $303 \mathrm{~K}$ with the $\mathrm{Cl} \cdots \mathrm{HO}$ correlation distance $(2.1 \AA$, coordination number 0.7 ) much shorter than that for $-\mathrm{CH}_{3} \cdots \mathrm{Cl}$ contacts between ions (observed at 2.8 A). Stefanovic et al. ${ }^{16}$ described correspondingly short $\mathrm{Cl}-\mathrm{HO}$ distances from simulation, however Wagle, Deakyne, and Baker ${ }^{15}$ found a longer correlation distance of $2.3 \AA$.

Here we observe the choline hydroxyl to chloride $\mathrm{Cl} \cdots \mathrm{HO}$ correlation at $338 \mathrm{~K}$ as a peak with a maximum at $2.8 \AA$ (coordination number of $0.80 \pm 0.84$ ), comparable in length to all the other choline $\mathrm{H} \cdots \mathrm{Cl}$ correlations found at $2.9 \AA$. This is a significant lengthening of the $\mathrm{Cl} \cdots \mathrm{HO}$ correlation distance and indicates a weakening of this structure directing association.

A second temperature effect is evident in the urea-urea (NU-HU) correlation, where a $\mathrm{N}-\mathrm{H} \cdots \mathrm{N}$ correlation with a sharp, well defined peak at $1.8 \AA\left(N_{\text {coord }}=1.98\right)$ can be observed. This is not present at $303 \mathrm{~K}$ where $\mathrm{N}-\mathrm{H} \cdots \mathrm{OU}$ urea self-association modes are more dominant.

Comparing with the literature, Stefanovic et al. ${ }^{16}$ reported weaker, and fewer $\mathrm{N}-\mathrm{H} \cdots \mathrm{OU}$ correlations and also a correspondingly larger coordination number for proximal $\mathrm{N}-\mathrm{H} \cdots \mathrm{Cl}$ interactions than found by Hammond, Bowron, and Edler ${ }^{11}$ in their EPSR analysis of neu- 
tron scattering data. Ashworth et al. ${ }^{14}$ noted the importance of $\mathrm{N}-\mathrm{H} \cdots \mathrm{N}$ hydrogen-bonding in ChCl:U, observing that $\mathrm{N}-\mathrm{H} \cdots \mathrm{N}$ interactions are equal to the stronger of the two $\mathrm{N}-\mathrm{H} \cdots \mathrm{O}$ associations in distorted hydrogen-bonded urea chains. Araujo et al. ${ }^{19}$ suggested that ureaurea $\mathrm{N}-\mathrm{H} \cdots \mathrm{O}$ interactions were not strengthened in $\mathrm{ChCl}: \mathrm{U}$, making the point that an increase in the (conventional) hydrogen-bonding interactions between urea molecules would lead to an increase in melting point. However, they also propose the formation of a centrosymmetric urea dimer, held by strong hydrogen bonds.

Together, the differences in the choline-OH $\cdots$ chloride and urea $\mathrm{N}-\mathrm{H} \cdots \mathrm{N}$ pRDFs suggest reorganisation in the liquid leading to a loss of the short distance choline hydroxyl to chloride correlations and a change in urea-urea self-association and loss of directional correlations to choline cations.

\section{Structure of ChCl:Ox DES}

Having established that the changes observed for $\mathrm{ChCl}$ : $\mathrm{U}$ can be understood principally in terms of a reduction in short distance correlations between choline and both Cl- and $\mathrm{U}$, we turn to $\mathrm{ChCl}$ :Ox to examine the influence of changing the HBD.

Zahn, Kirchner, and Mollenhauer ${ }^{28}$ have studied the effects of HBD components on the magnitude of interactions in the DES by DFT modelling, predicting an increase in HBD $\cdots$ chloride hydrogen-bonding from urea (through ethylene glycol) to oxalic acid as a HBD. This is supported by polarity studies ${ }^{30}$ that show DES containing hydroxyl-containing HBD donating components were more polar that those containing urea.

Choline $\mathrm{C}-\mathrm{H} \cdots \mathrm{Cl}$ pRDF correlations in $\mathrm{ChCl}$ :Ox (Figure 4) cluster at 2.7-2.8 $\AA$, with the $\mathrm{N}-\mathrm{Cl}$ correlation showing a maxima at $4.7 \AA$, similar to those found in ChCl: $\mathrm{U}$, underlining the similarities observed in the COM RDFs in Figure 3. In both DES, the $\mathrm{N}-\mathrm{Cl} \mathrm{pRDF}$ shows evidence of two overlapping peaks which reflect the two closest approaches of chloride to the N-centre via the N-ethyl and N-methyl substituents. The choline-OH $\cdots$ Cl correlation is also found with a maximum at $2.8 \AA$ (coordination number 0.94 ), the same distance as that for the first peaks in the choline $\mathrm{C}-\mathrm{H} \cdots \mathrm{Cl}$ pRDFs indicating that there is a significant reduction in the hydrogen-bonding interactions with the choline hydroxyl group at $338 \mathrm{~K}$.

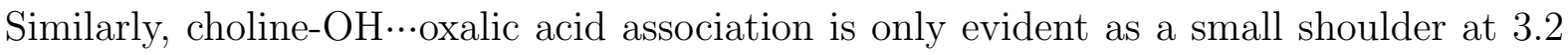
$\AA$ (to a minimum at $4.1 \AA$ ) in the O1-OA2 correlation. Hydrogen-bonding between the 
choline hydroxyl site and oxalic acid can occur with either choline or oxalic acid acting as

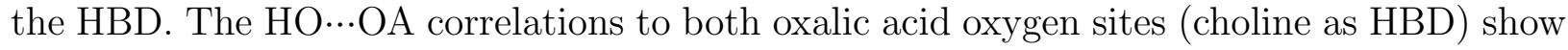

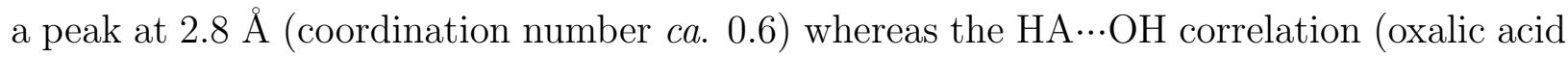
as HBD) has no distinct peak, but the contact starts from $1.7 \AA$. The coordination number,

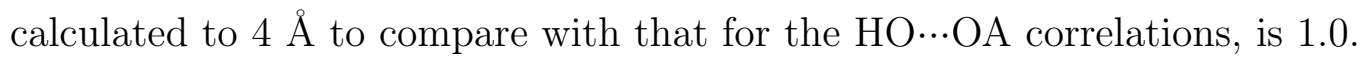

Strong association of the oxalic acid with chloride anions is evident from the peaks at 2.0 $\AA$ and $2.9 \AA$ in the $\mathrm{HA}-\mathrm{Cl}$ and $\mathrm{Cl}-\mathrm{OA}$ pRDFs respectively. This is comparable to the HBD$\mathrm{Cl}$ pRDFs observed previously with peaks around 2.0-2.2 $\AA$ in ChCl:U and ChCl:Mal. ${ }^{11,12}$ The HA-Cl peak distance found is consistent with the value of $2.95 \AA$ obtained from DFT calculations ${ }^{28}$ and is slightly shorter than the corresponding $\mathrm{ChCl:U} \mathrm{Cl-NU}$ correlation at $3.0 \AA$. The $\mathrm{Cl} \cdots \mathrm{X}$ separation ( $\mathrm{X}=$ hetero-atoms of the HBD, $\mathrm{N}$ or $\mathrm{O}$ ) decreases from 3.35 $\AA$ in $\mathrm{ChCl}: \mathrm{U}$ to $2.95 \AA$ in $\mathrm{ChCl}: \mathrm{Ox}$ while the choline- $\mathrm{OH} \cdots \mathrm{Cl}$ interaction distance remained approximately constant at $3.1 \AA(\mathrm{O} \cdots \mathrm{Cl})$. An increase in HBD-chloride hydrogen-bond strength on changing the DES components from urea to polyols and carboxylic acids was also found from QENS $^{9}$ and is supported by polarity studies. ${ }^{30}$

Self-association of oxalic acid is evident from the peak at $5.0 \AA$ in the COM RDF in

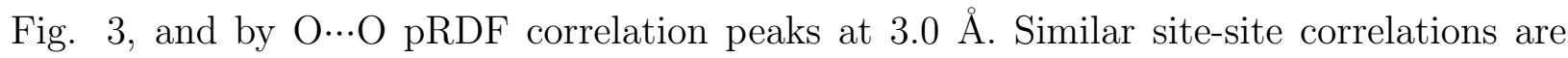

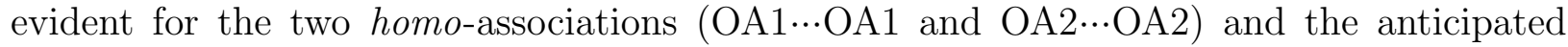

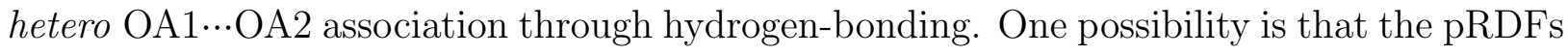
incorporates elements of discrete acid-acid hydrogen-bonding and spatial proximity that occurs through interactions of oxalic acids with chloride anions. The OA2 $\cdots \mathrm{HA}$ acid-acid hydrogen bonding correlation starts at $1.5 \AA$ and extending up to $c a .2 .2 \AA$, however there is no distinct peak.

This contrasts with pure acetic and formic acid ${ }^{31}$ and also pyridine/acetic acid mixtures ${ }^{32}$ where strong acid $\cdots$ acid hydrogen-bonding is observed. This suggests that a significant proportion of the $\mathrm{pRDF}$ represents proximal association rather than specific hydrogen-bonding correlations.

In terms of the HBD-chloride and self-association (urea-urea or oxalic acid-oxalic acid), there are marked differences in the structure correlations between the two DES. Strong HBD - chloride correlations are apparent at $2.0 \AA$ in both $\mathrm{ChCl:U}$ and $\mathrm{ChCl}: \mathrm{Ox}$. Although the position of these peaks are equivalent in both DES (contrasting with small reduction in separation predicted by Zahn, Kirchner, and Mollenhauer ${ }^{28}$ from $\mathrm{ChCl:U}$ to $\mathrm{ChCl:Ox}$ ), 
the peak is noticeably sharper in $\mathrm{ChCl}: \mathrm{Ox}$. The coordination numbers for chloride ions to either urea-HU or oxalic acid-HA sites are 0.175 and 0.275 respectively, calculated to 2.5 $\AA$, and 0.309 and 0.438 calculated to $3 \AA$, showing greater solvation of chloride by oxalic acid in $\mathrm{ChCl:Ox}$ than by urea in $\mathrm{ChCl:U}$. The hydrogen-bond donor:chloride ratio in the two DES are 8:1 (ChCl:U) and 2:1 (ChCl:Ox) providing evidence for the highly correlated chloride-oxalic acid liquid structure.

\section{Spatial Density Functions}

Spatial distributions (SDFs) of choline, chloride and either urea or oxalic acid around both choline and urea/oxalic acid centres in the two DES are shown in Figure 5. Both DES show comparable distributions of components around the choline cations using the molecular centres of mass as the point of reference.

The choline-centred SDFs reveal that chloride has the closest interaction with choline, ordering at the contact distance (4.6-4.7 $\AA$ ) with the highest correlation probability as a band around the hydroxyethyl-group. Further association at the same distance to the methylgroups of the cation can also be seen. The isosurface is large and relatively diffuse covering a large proportion of the available space around choline through ion-ion interactions. The flexibility of the $\mathrm{NCH}_{2} \mathrm{CH}_{2} \mathrm{OH}$ group and the large conformational volume that this group can occupy through rotations mean that a detailed assessment of the spatial correlations is necessarily limited by the selection of the reference point for observation.

The urea and oxalic acid SDFs around choline in the respective DES have similar profiles to those of chloride at slightly longer correlation distances (as indicated in the COM RDFs, Fig. 3) with similar spatial distributions. The choline-choline solvation shell appears at larger distances and are also largely associated with the positions of the chloride (and HBD) in a band about the central choline, demonstrating the radially layering evident from the COM RDFs.

In contrast to the broad, diffuse SDFs around choline, the HBD centred SDFs in Fig. 3 show much greater spatial organisation. The strong urea $\cdots \mathrm{Cl}$ association through the urea proximal hydrogen positions in $\mathrm{ChCl:U}$ is anticipated given the role of urea as an anion receptor. ${ }^{33}$ In contrast, urea-urea correlations appear through interactions with all four hydrogens. The strong HU-NU correlation in the pRDF (1.8 $\AA$ ) and urea-urea SDF 
Choline-urea and choline-oxalic acid DES

show development of amine-amine hydrogen-bonding networks rather than head-to-tail urea stacking through association with the urea-OU position. There is no evidence for correlation density associated with the OU position, at this moderately high probability $15 \%$ spatial density function, that would correspond to the NU-HU...OU associations.

These chlorine-urea and urea-urea spatial associations dominate the structure around urea, with choline cations occupying the remaining vacant space over the urea face and around the urea carbonyl group that has a reduced HBA role than that found at $303 \mathrm{~K}^{11}$ where chloride is associated with both urea distal and proximal hydrogen positions and urea-urea correlations were predominantly found associated with the distal N-H positions.

For ChCl:Ox, SDFs around oxalic acid (Fig. 5) show a highly symmetric distribution forming a band around the CA-CA bond and, in the molecular plane, lobes extending from each $-\mathrm{CO}_{2} \mathrm{H}$ function. The chloride SDF strong correlation as a band around the $\mathrm{CA}-\mathrm{CA}$ bond with single-site hydrogen-bonding to the two carboxylic acid hydrogen sites (seen as the vertically arranged lobes in Figure 5 and bridging 'side-on' to the oxalic acid). Asymmetry present in the SDF profile may reflect limitations in the static EPSR reference potential since vibration spectroscopy shows that the two carboxylic acid O-sites are equivalent due to rapid hydrogen-exchange. Similarly the oxalic acid-oxalic acid SDF presents a band of high correlation probability around the CA-CA bond (without the asymmetry lobes) and two lobes from each $-\mathrm{CO}_{2} \mathrm{H}$ group. When SDFs are calculated using a larger probability sample (not shown) the lobes merge into two concentric bands bisecting the plane of the molecule. This distribution of states appears consistent with the large number of inter- and intra-molecular hydrogen-bonding modes and $\pi-\pi$ stacking interactions possible in oxalic acid ${ }^{34}$ with the most stable dimers containing two inter-molecular and three intramolecular hydrogen-bonds (Figure 6).

The correlation band surrounding the CA-CA bond is common to both the oxalic acidoxalic acid and oxalic acid-chloride SDFs, and is composed of hydrogen-bonding from intramolecularly-constrained oxalic acid hydrogen atoms to chloride (in the plane) and to presumably anion- $\pi$ out-of-plane interactions. Terminal hydrogen-bonds between oxalic acid and chloride result in the asymmetric lobes, whereas the generation of two hydrogen bonds between oxalic acid pairs (each acid as an acceptor and a donor) leads to the linear correlation sites. The choline-oxalic acid SDF also maps to the oxalic acid-oxalic acid correlations, but at a longer separation distance due to the larger size of the cation. The largest struc- 
Choline-urea and choline-oxalic acid DES

tural association around the oxalic acid groups are those arising from purely inter-molecular $\mathrm{COOH} \cdots \mathrm{Cl}$ bonds producing the chloride-lobes that point to strong and persistent hydrogenbonding.

Strong choline-OH$\cdots \mathrm{Cl}$ hydrogen-bonding was reported as the dominant ordering interaction in $\mathrm{ChCl}: \mathrm{Mal}^{12}$ with hydrogen bonding to chloride from both the acid and alcohol sites (coordination numbers of 0.62 and 0.51 respectively) and the alcohol-OH $\cdots \mathrm{Cl}$ as the preferred interaction at $303 \mathrm{~K}$. In contrast, here at $338 \mathrm{~K}$, with the loss of choline-OH correlations in the DES and the stronger $\mathrm{HBD}$ component, $\mathrm{ChCl:Ox}$ exhibits a more correlated structure. Charge delocalisation and transfer from chloride to oxalic $\operatorname{acid}^{28}$ leads to the hydrogen bonding competition between oxalic acid-chloride and oxalic acid-oxalic acid. A common theme between $\mathrm{ChCl}: \mathrm{Ox}$ (here) and $\mathrm{ChCl}: \mathrm{Mal}$ is the presence of carboxylic acid molecules oriented radially in parallel with the long axis of a central acid. However, direct comparisons are hindered by the differences in pKa (1.23 for oxalic acid, 2.83 for malic acid) and the increased conformational flexibility and the presence of an additional -OH group in malic acid.

\section{Validation of EPSR reference potential}

The results obtained for $\mathrm{ChCl}$ : $\mathrm{U}$ show some significant differences in the specific site-site correlations present in the DES compared to the structure previously described at $303 \mathrm{~K}^{11}$ In particular, the key loss in choline-chloride structure and changes to the urea-urea correlations. It is important to ensure that this accurately reflects reasonable solutions constrained in the refinement by the experimental data rather than being perturbed or defined by the initial reference potential.

Hammond, Bowron, and Edler ${ }^{11}$ used a model for their data refinement that differed from that here by the use of (i) integer \pm 1 charges assigned to the $N$-center of choline and the chloride anion, and (ii) a relatively high +0.6 charge placed on the hydroxyl-hydrogen of choline in the EPSR reference potential. In this work, charge on choline was distributed over the four carbon centres attached to the N-atom and a smaller overall partial charge was assigned to the hydroxyl-group making it less acidic (see Table I). To test whether these differences in the reference potential could lead to undesired bias in the final refinement, particularly generating potentially stronger $\mathrm{HO}-\mathrm{Cl}$ interactions at shorter distances, we re- 
Choline-urea and choline-oxalic acid DES

refined the $\mathrm{ChCl}: \mathrm{U}$ data here using the earlier reference potential. After equilibration, the refined structure model obtained showed no change on either peak positions or coordination numbers associated with RDFs to the choline-OH sites, and we can conclude that the refinement is robust and that the losses in the specific site-site correlations with the choline-OH sites are due to increased thermal motion and, probably, rotation of the choline cation.

\section{CONCLUSIONS}

The key observation from the investigation of $\mathrm{ChCl}: \mathrm{U}$ and $\mathrm{ChCl}: \mathrm{Ox}$ DES at $338 \mathrm{~K}$ is that the choline-hydroxyl group does not act as a structure directing site in the liquids. All the key short-length associations of choline; choline- $\mathrm{OH} \cdots \mathrm{Cl}$ and self-association of choline cations through $\mathrm{HO}-\mathrm{OH}$ hydrogen-bonding, previously reported around $2.0 \AA$, extended to $2.8 \AA(\mathrm{HO} \cdots \mathrm{Cl})$ and $3.0 \AA(\mathrm{HO} \cdots \mathrm{OH})$.

Changing the EPSR reference potential to match that from previous studies at $303 \mathrm{~K}^{11,12}$ did not produce a better refinement fit, and consequently we can conclude that the models generated are equally consistent with the experimental data at these moderately elevated temperatures, and describe liquid structure in both $\mathrm{ChCl}: \mathrm{U}$ and $\mathrm{ChCl}: \mathrm{Ox}$ DES in which the choline-OH group plays a more less prominent role as either a hydrogen-bond donor or acceptor compared to that previously reported at $303 \mathrm{~K}$.

An alternative explanation, that the DES all consistently contain water that was not considered in the modelling and could interfere with choline-OH $\cdots$ anion hydrogen bonding is inconsistent with both the physical behaviour of the DES samples (melting points) and the good match of neutron scattering cross section to the anticipated material compositions. Moreover, it has reported that addition of 1 mole of water in $\mathrm{ChCl:U} \mathrm{DES}^{35}$ produced a strengthening of the choline- $\mathrm{OH} \cdots \mathrm{H}_{2} \mathrm{~N}$ hydrogen-bonding interaction in contrast to the reduction in interactions observed here as the temperature in increased.

DFT modelling of ChCl-containing DES with urea, ethylene glycol and glycerolStefanovic et $a l .{ }^{16}$ have shown correlations between the choline ammonium-charge centre to the HBD (characterised by a broad correlation around $4 \AA$ ) and HBD ... Cl interactions (around $2.0 \AA$ ) were dominant structural features. They also concluded that choline-OH$\cdots \mathrm{Cl}$ hydrogen bonding was less pronounced than HBD … Cl hydrogen bonding (consistent with Wagle, Baker, and Mamontov $^{9}$ ) and that this, therefore, is not a key structural interaction. The 
Choline-urea and choline-oxalic acid DES

systematic loss of short-range (and by implication, strong) choline-OH to chloride correlations in both $\mathrm{ChCl}: \mathrm{U}$ and $\mathrm{ChCl}: \mathrm{Ox}$ studied here at $338 \mathrm{~K}$ is most likely a result of increasing rotational mobility of the choline cation which becomes increasingly a spectator cation as the temperature increases. As such, the hydroxyl group in choline does not appear to be a key structural feature for DES formation which is consistent with the observation that DES can be readily formed with many symmetric organic salts. ${ }^{2}$

\section{ACKNOWLEDGMENTS}

The authors gratefully acknowledge funding and support from the Department for Employment and Learning, Northern Ireland (to AHT), Queen's University Ionic Liquids Laboratory (to MG), European Union's Horizon 2020 research and innovation programme (grant agreement No. 655334 to LM), EPSRC (departmental PhD studentship EP/K503009/1 to JAM) and the STFC for beam-time (RB1620018 and RB1700022) at the ISIS Pulsed Neutron and Muon Source.

\section{REFERENCES}

${ }^{1}$ A. P. Abbott, G. Capper, D. L. Davies, R. K. Rasheed, and V. Tambyrajah, Chem. Comm. , 70 (2003).

${ }^{2}$ A. P. Abbott, G. Capper, D. L. Davies, H. L. Munro, R. K. Rasheed, and V. Tambyrajah, Chem. Commun., 2010 (2001).

${ }^{3}$ A. P. Abbott, D. Boothby, G. Capper, D. L. Davies, and R. K. Rasheed, J. Am. Chem. Soc. 126, 9142 (2004).

${ }^{4}$ A. P. Abbott, ChemPhysChem 5, 1242 (2004).

${ }^{5}$ Q. Zhang, K. De Oliveira Vigier, S. Royer, and F. Jérôme, Chem. Soc. Rev. 41, 7108 (2012).

${ }^{6}$ M. Francisco, A. vandenBruinhorst, and M. C. Kroon, Angew. Chem. Int. Ed. 52, 3074 (2013).

${ }^{7}$ C. D’Agostino, R. C. Harris, A. P. Abbott, L. F. Gladden, and M. D. Mantle, Phys. Chem. Chem. Phys. 13, 21383 (2011). 
Choline-urea and choline-oxalic acid DES

${ }^{8}$ A. P. Abbott, R. C. Harris, K. S. Ryder, C. D'Agostino, L. F. Gladden, and M. D. Mantle, Green Chem. 13, 82 (2011).

${ }^{9}$ D. V. Wagle, G. A. Baker, and E. Mamontov, J. Phys. Chem. Lett. 6, 2924 (2015).

${ }^{10}$ O. Russina, S. De Santis, and L. Gontrani, RSC Adv. 6, 34737 (2016).

${ }^{11}$ O. S. Hammond, D. T. Bowron, and K. J. Edler, Green Chem. 18, 2736 (2016).

${ }^{12}$ O. S. Hammond, D. T. Bowron, A. J. Jackson, T. Arnold, A. Sanchez-Fernandez, N. Tsapatsaris, V. Garcia Sakai, and K. J. Edler, J. Phys. Chem. B 121, 7473 (2017).

${ }^{13}$ S. L. Perkins, P. Painter, and C. M. Colina, J. Chem. Eng. Data 59, 3652 (2014).

${ }^{14}$ C. R. Ashworth, R. P. Matthews, T. Welton, and P. A. Hunt, Phys. Chem. Chem. Phys. 18, 18145 (2016).

${ }^{15}$ D. V. Wagle, C. A. Deakyne, and G. A. Baker, J. Phys. Chem. B 120, 6739 (2016).

${ }^{16}$ R. Stefanovic, M. Ludwig, G. B. Webber, R. Atkin, and A. J. Page, Phys. Chem. Chem. Phys. 19, 3297 (2017).

${ }^{17}$ S. Zahn, Phys. Chem. Chem. Phys. 19, 4041 (2017).

${ }^{18}$ E. L. Smith, A. P. Abbott, and K. S. Ryder, Chem. Rev. 114, 11060 (2014).

${ }^{19}$ C. F. Araujo, J. A. P. Coutinho, M. M. Nolasco, S. F. Parker, P. J. A. Ribeiro-Claro, S. Rudic, B. I. G. Soares, and P. D. Vaz, Phys. Chem. Chem. Phys. 19, 17998 (2017).

${ }^{20}$ A. H. Turner and J. D. Holbrey, J. Solution Chem. 44, 621 (2015).

${ }^{21}$ C. Hardacre, J. D. Holbrey, C. L. Mullan, M. Nieuwenhuyzen, T. G. A. Youngs, D. T. Bowron, and S. J. Teat, Phys. Chem. Chem. Phys. 12, 1842 (2010).

${ }^{22}$ M. C. Gutierrez, D. Carriazo, C. O. Ania, J. B. Parra, M. Luisa Ferrer, and F. del Monte, Energy \& Environ. Sci. 4, 3535 (2011); N. Lopez-Salas, D. Carriazo, M. C. Gutierrez, M. L. Ferrer, C. O. Ania, F. Rubio, A. Tamayo, J. L. G. Fierro, and F. del Monte, J. Mat. Chem. A 4, 9146 (2016); O. S. Hammond, K. J. Edler, D. T. Bowron, and L. Torrente-Murciano, Nature Comm. 8, 14150 (2017).

${ }^{23}$ X. Meng, K. Ballerat-Busserolles, P. Husson, and J.-M. Andanson, New J. Chem. 40, $4492(2016)$.

${ }^{24}$ A. K. Soper, RAL Tech. Rep. RAL-TR-201 (2011).

${ }^{25}$ A. K. Soper, Chem. Phys. 202, 295 (1996).

${ }^{26}$ A. K. Soper, Mol. Phys. 99, 1503 (2001).

${ }^{27}$ A. K. Soper, Mol. Phys. 107, 1667 (2009).

${ }^{28}$ S. Zahn, B. Kirchner, and D. Mollenhauer, ChemPhysChem 17, 3354 (2016). 
Choline-urea and choline-oxalic acid DES

${ }^{29}$ C. Hardacre, J. D. Holbrey, M. Nieuwenhuyzen, and T. G. A. Youngs, Acc. Chem. Res. 40, 1146 (2007).

${ }^{30}$ A. Pandey, R. Rai, M. Pal, and S. Pandey, Phys. Chem. Chem. Phys. 16, 1559 (2014).

${ }^{31}$ S. Imberti and D. T. Bowron, J. Phys. Cond. Mat. 22, 404212 (2010).

${ }^{32}$ J. A. McCune, A. H. Turner, F. Coleman, C. M. White, S. K. Callear, T. G. A. Youngs, M. Swadźba-Kwaśny, and J. D. Holbrey, Phys. Chem. Chem. Phys. 17, 6767 (2015).

${ }^{33}$ V. Amendola, D. Esteban-Gómez, L. Fabbrizzi, and M. Licchelli, Acc. Chem. Res. 39, 343 (2006); V. Amendola, L. Fabbrizzi, and L. Mosca, Chem. Soc. Rev. 39, 3889 (2010). ${ }^{34}$ S. A. Blair and A. J. Thakkar, Chem. Phys. Lett. 495, 198 (2010).

${ }^{35}$ O. S. Hammond, D. T. Bowron, and K. J. Edler, Angew. Chem. Int. Ed. 56, 9782 (2017). 
Choline-urea and choline-oxalic acid DES
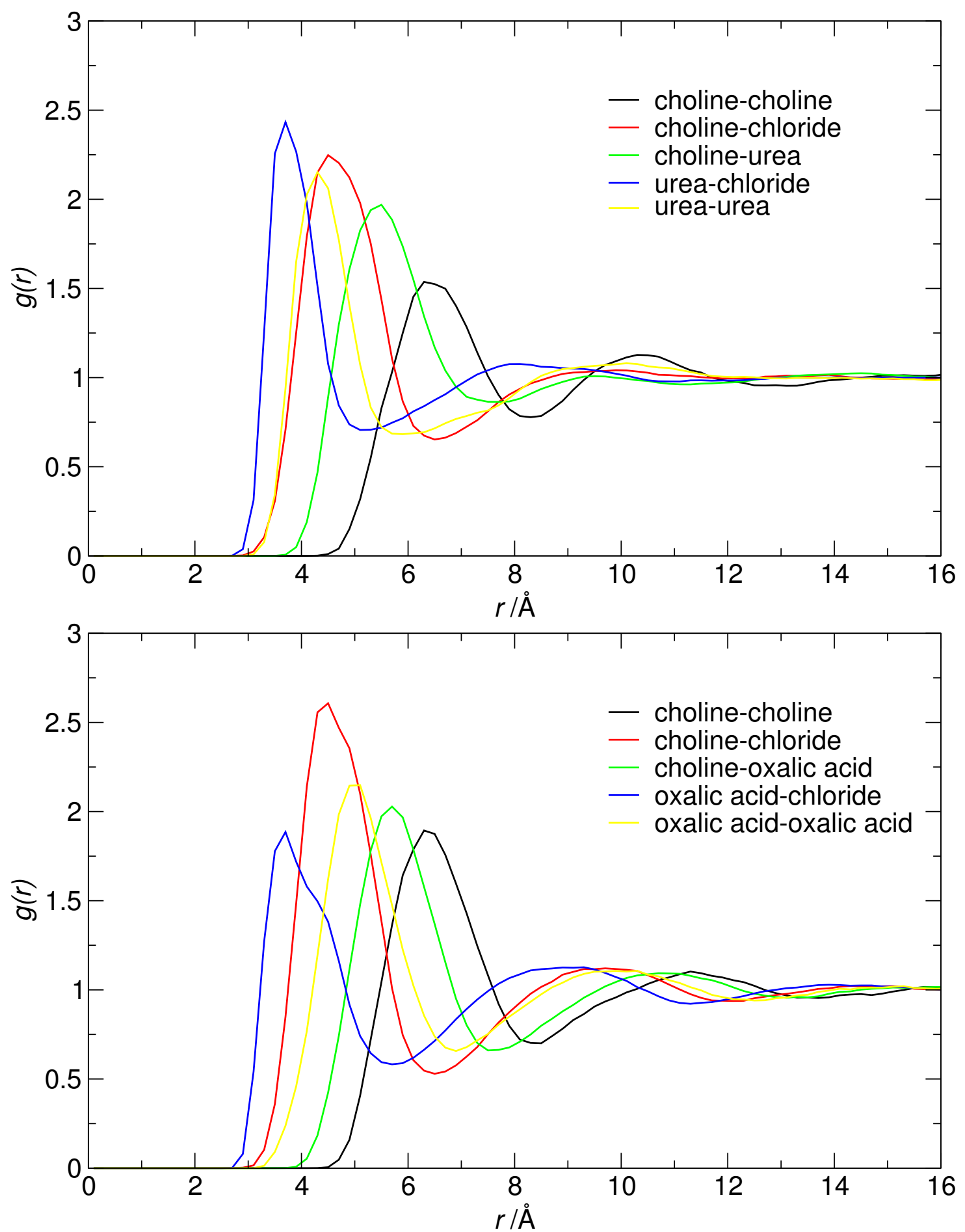

Figure 3. Centre of mass RDFs for $\mathrm{ChCl}: \mathrm{U}$ (top) and $\mathrm{ChCl:Ox}$ (bottom). 
Choline-urea and choline-oxalic acid DES
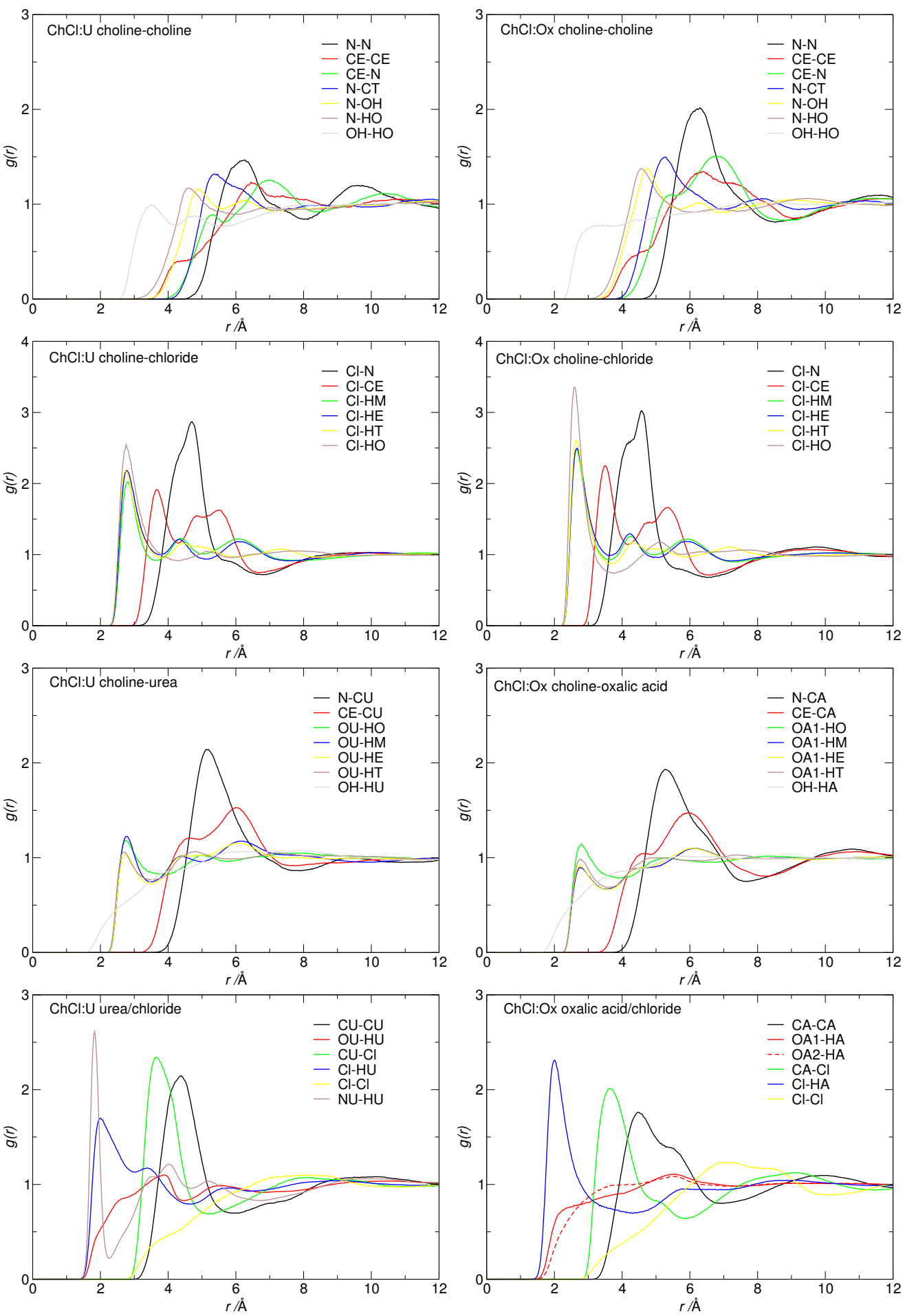

Figure 4. Atom-centred pRDFs for $\mathrm{ChCl:U}$ (left) and $\mathrm{ChCl:Ox}$ (right). 

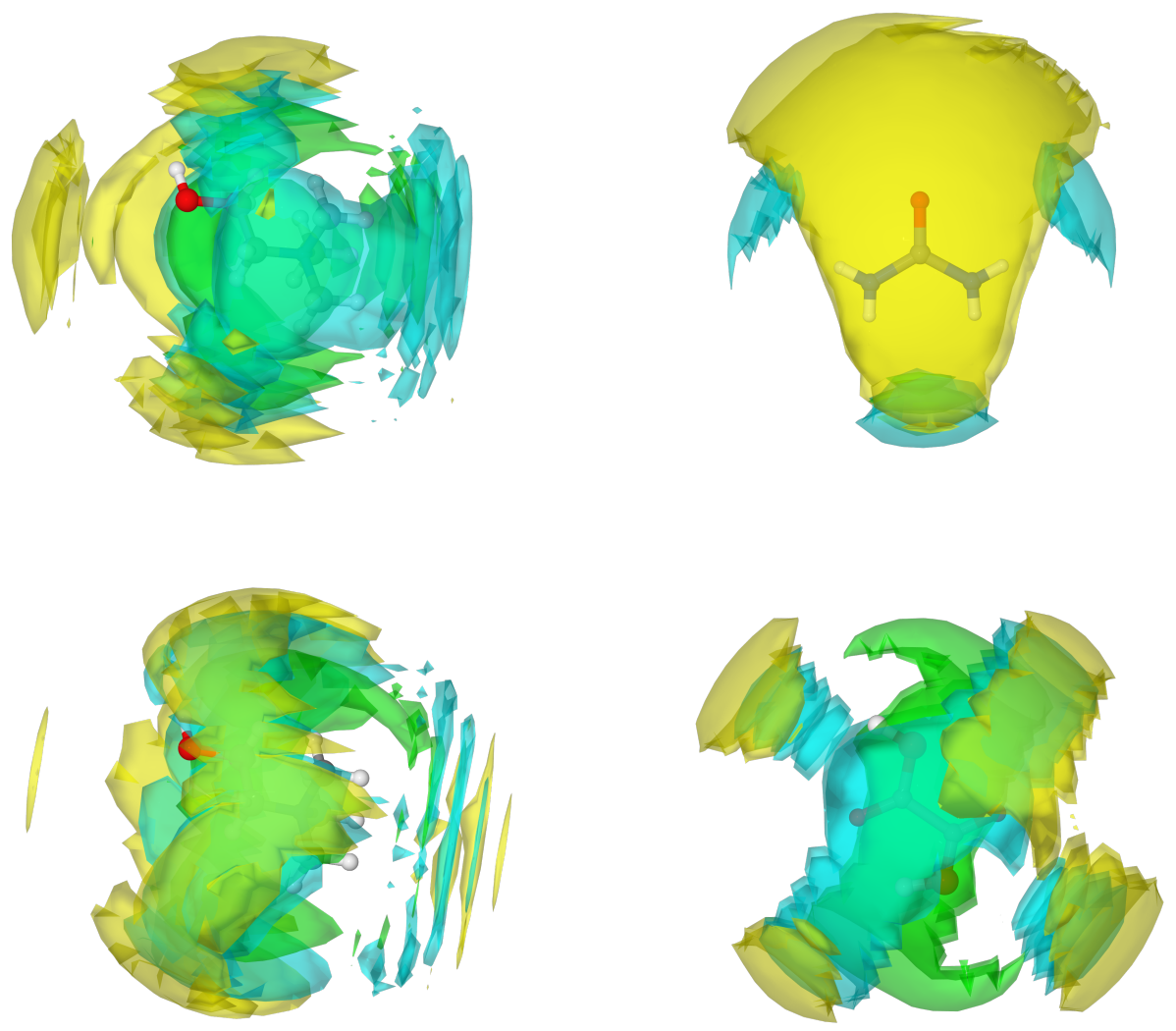

Figure 5. SHARM spatial probability maps for $\mathrm{ChCl:U}$ (top) and $\mathrm{ChCl:Ox}$ (bottom) showing the distributions around choline in the two systems (left) and urea (top right) or oxalic acid (bottom right) of choline (yellow), chloride (green) and urea or oxalic acid (cyan) calculated to encompass the first shell peaks from the COM RDFs in Figure 3. Surfaces were calculated to encompass the top $15 \%$ probability within the first peak in the COM RDF around the central molecule. 


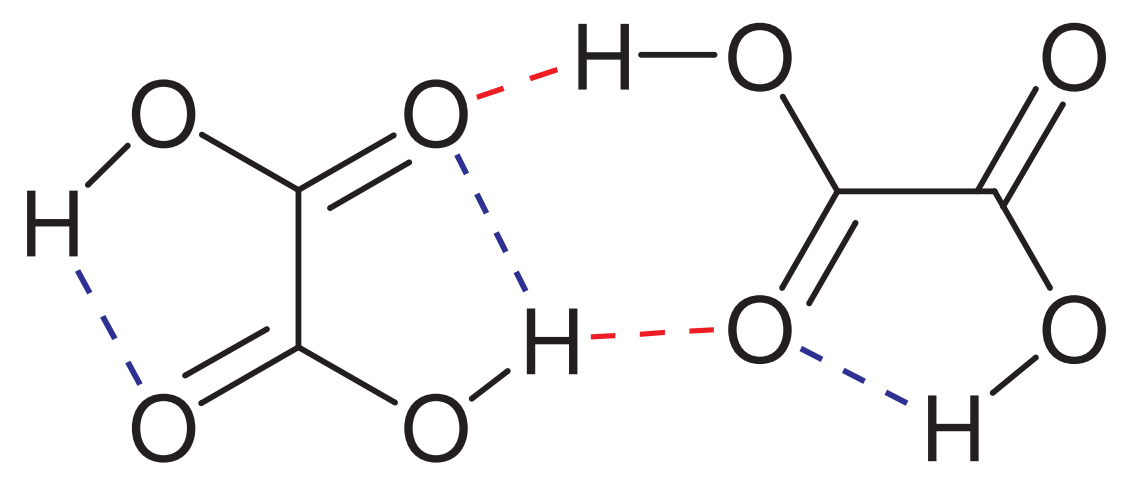

Figure 6. Schematic of the most favourable oxalic acid dimer hydrogen-bonding motif (after Blair and Thakkar ${ }^{34}$ ) with two inter-molecular hydrogen-bonds (red) and three intra-molecular hydrogen-bonds (blue) that generate the characteristic pattern in the oxalic acid-oxalic acid SDF 